DISKUSSIONSFORUM

\section{Entlastung vs. Entmündigung}

\author{
Assistenz- und Pflegesysteme in Zeiten \\ des demographischen Wandels
}

\section{von Klaus Wiegerling, ITAS}

Es wird keine humane Abfederung der Probleme einer alternden Gesellschaft geben ohne die Nutzung assistiver Systeme. Der Zeitpunkt, die notwendigen Pflege- und Assistenzleistungen für Alte, Behinderte und Kranke personell noch in den Griff zu bekommen, scheint verpasst zu sein. Die demographische Lawine ist ins Rollen gekommen. Es wäre aber ein Irrtum zu glauben, dass assistive Systeme allein durch soziale Notwendigkeiten initiiert sind. Ihre Entwicklung ist im Kontext der informatischen Idee der „Ambient Intelligence“ zu sehen. Die ganze Handlungsumgebung soll intelligent werden und uns bei unseren Alltagsverrichtungen unterstützen. Die Bedienungsanforderungen sollen minimal sein bzw. durch automatisierte Prozesse ersetzt werden. Das System soll uns unsere Wünsche quasi von den Lippen ablesen und uns in vorauseilendem Gehorsam dienen.

Die Systementwicklung ist aber auch ökonomisch initiiert. Rationalisierungsmöglichkeiten und damit auch wirtschaftliche Effizienzsteigerungen sollen geboten werden. Die Entwicklung vernetzter verteilter Systeme, die Handlungssituationen verstehen, sich nutzeradaptiv verhalten und die Mensch-System-Schnittstelle weitgehend zum Verschwinden bringen, bietet in allen Bereichen des Gesundheitswesens Anwendungsmöglichkeiten. Mit Hilfe solcher Systeme lassen sich extra- und intrakorporal Gesundheitszustände überwachen. Intrakorporal sind über intelligente Implantate Steuerungen physiologischer Prozesse möglich. Man könnte etwa durch ein Implantat nicht nur permanent die Blutzuckerwerte eines Diabetikers erheben, sondern über ein Depot entsprechend der festgestellten Zuckerwerte Insulin abgeben, ohne dass der Patient ständig die Werte überprüfen und sich spritzen muss.
Durch informatische Entwicklungen, die unter Stichworten wie Biocybernetic oder Neurophysiological Computing gefasst werden, wird zudem der Anspruch erhoben, auch Stimmungen, emotionale Zustände, ja sogar Intentionen von Nutzern durch Brain-Reading erkennen und für die Interaktion mit dem System nutzen zu können. Das heißt, das Assistenzsystem würde nicht nur unsere physiologischen Zustände, sondern auch die psychischen überwachen; und es würde selbständig auf diese Zustände reagieren, uns etwa aufheiternde Medikamente anbieten oder einen Telefonkontakt mit einem Freund herstellen.

\section{Assistenzsysteme als Unterstützungs- und Entlastungssysteme}

Die Technologie, in die die Entwicklung assistiver Systeme eingebettet ist, versucht die gesamte Mesosphäre informatisch zu erschließen. Jeder Gegenstand in ihr kann unser Kommunikationspartner und Informant werden und uns dienstbar sein. Die Idee eines Ambient Assisted Living (AAL) geht also über die Unterstützung von Alten, Behinderten und Kranken hinaus. Assistive Systeme sollen alle, auch Junge, Gesunde und Nichtbehinderte jederzeit und überall begleiten und uns im gesamten Alltagsleben unterstützen. Die Grenzen zwischen der Assistenz des Alltagslebens und der Pflege- oder Behindertenassistenz sollen fließend sein. Während es im einen Fall nur um Entlastung bei Alltagsgeschäften geht, geht es im anderen Fall auch um die Ermöglichung der Teilnahme an sozialen Prozessen.

Entlastet werden soll auch das Umfeld Alter, Kranker und Behinderter, nicht zuletzt die Gesellschaft. Zynischerweise könnte man sagen, dass auch eine Gewissensberuhigung von Angehörigen und Gesellschaft stattfindet, wenn AAL-Systeme mitmenschliche Verpflichtungen übernehmen. Doch nur so ist die Pflege und regelmäßige Unterstützung $\mathrm{zu}$ gewährleisten. Gründe sind der genannte demographische Wandel, begrenzte ökonomische Ressourcen, berufliche Verpflichtungen von Angehörigen, aber auch gesellschaftliche Entwicklungen, die die klassische Gesellschaftsformierung in Familienverbänden und nichtanonymen Nachbarschaftsbeziehungen hinter sich gelassen haben. Man mag dies bedauern, aber die 
Vorstellung einer humanen Gesellschaft, die sich in dauerhafter wechselseitiger Unterstützung artikuliert, muss im Reich der Sehnsüchte angesiedelt werden. Eine gewisse Isolation und soziale „Reduzierung“ des Lebens von Alten und Pflegebedürftigen wird sich beim vermehrten Einsatz assistiver Systeme kaum vermeiden lassen. Andererseits wird es aber auch mediale Vernetzungsmöglichkeiten dieser Personen und damit auch gewisse Kompensationen der sozialen Reduktion geben.

AAL soll das alltägliche Leben unaufdringlich unterstützen. Die Technologie soll nutzerzentriert sein, wobei man sinnvollerweise zwischen Nutzer und Nutznießer unterscheiden sollte. Nutznießer soll letztlich der zu Pflegende bzw. zu Unterstützende sein, ob er das System aktiv steuernd nutzt oder nicht. Nutzer können natürlich auch Pflegekräfte, Ärzte und Angehörige sein, die in einem nachgeordneten Sinne Nutznießer sind. Pflegebedürftige sollen nicht nur bei alltäglichen hygienischen Verrichtungen unterstützt, ihr Gesundheitsstatus soll auch überwacht werden mit der Option auf einen automatisierten Eingriff in den organischen Zustand mittels intelligenter Implantate. Zuletzt soll eine Integration des Unterstützten in sein soziales Umfeld über mediale Technologien geleistet werden. Die adaptive Technologie soll sich an die Gewohnheiten, Zustände und Intentionen des Nutzers anpassen. Dessen Lebensqualität soll eine Steigerung erfahren, alten und multimorbiden Menschen ein längerer Aufenthalt in der gewohnten Umgebung ermöglicht werden. Die umfassende Observation des äußeren und inneren Zustandes soll die Angst vor Isolation und Hilflosigkeit nehmen, die Vernetzung mit medizinischen und pflegerischen Einrichtungen soll gewährleistet, eine soziale Ausgrenzung vermieden werden.

\section{Entlastung als Grundprinzip der Anthropologie und Technikphilosophie}

Arnold Gehlen führte die Entlastung als Schlüsselkategorie in die moderne Anthropologie ein (Gehlen 1993). Er bestimmt den Menschen als biologisches Mängelwesen. Tiere übertreffen ihn an Instinktsicherheit und Sinnesschärfe. Gleichzeitig sind diese Mängel eine besondere Auszeichnung, denn der Mensch ist nicht in die Umwelt eingefügt und erweist sich, da er dennoch überlebensfähig ist, als das am höchsten organisierte Tier. Permanent zum Handeln gezwungen lässt der weltoffene Mensch den situationsgebundenen Spielraum des Tieres hinter sich. Schon Lernprozesse erweisen sich als Entlastungsfaktoren, mit deren Hilfe Handlungen unabhängig vom Reiz wechselnder Situationen ausgeführt werden können. Durch die Formung und Bändigung seines Antriebsüberschusses wird für den Menschen Energie verfügbar, die für die Gestaltung der Lebensbedingungen genutzt werden kann. So dienen niedrige wie höhere Vermögen der Lebensbewältigung, womit auch kulturelle Hervorbringungen in den Kontext der Lebenserhaltung gestellt sind. Der Mensch erschafft sich quasi selbst mit Hilfe von Sprache, Kultur, Erziehung und Werkzeugen. Durch die fortschreitende Mittelbarkeit des menschlichen Verhaltens, das nicht auf die Erfüllung primärer körperlicher Bedürfnisse, sondern auf die Ermöglichung einer entlastenden Infrastruktur gerichtet ist, die die Erfüllung dieser Bedürfnisse beständig gewährleistet, wird die Effektivität des Handelns erhöht. Gleichzeitig kommt es aufgrund neuer sekundärer Bedürfnisse zu Ablenkungen, Deformationen und Störungen. Dem Menschen, so Gehlen, ist die Vertauschung von Mittel und Zweck gelungen. Nicht das Befriedigungserlebnis wird angestrebt, sondern der Akt, bei dem es auftritt. Es findet eine Umkehrung der Antriebsrichtung statt. So kann der Mensch über seine Antriebe herrschen und es zu einer Selbststeigerung bringen. Die Notwendigkeit sich zu entlasten, birgt aber auch Gefahren. Gewohnheiten ketten uns an die Vergangenheit und machen uns träge. Selbst wenn die Technik den Menschen entlastet und dessen Überleben sichert, sieht Gehlen aber auch die Gefahr, dass in einer technisch hoch erschlossenen Welt ein Antriebsüberschuss entstehen kann, der sich aggressiv gegen die Mit- und Innenwelt richtet.

Das Entlastungsprinzip, das Gehlen in die moderne Anthropologie einführt, galt schon der frühen Technikphilosophie als Rechtfertigungsgröße. Für Ernst Kapp, der im 19. Jahrhundert die moderne Technikphilosophie begründete, muss der Mensch seine Mängel durch technische Projektionen kompensieren (Kapp 1877). Und für Friedrich Dessauer ist Technik die „Emanzipation des Menschen (...) aus der vegitabilen und animalischen Gebundenheit und Abhängigkeit 
(...). Technik bedeutet (...) Freiheit von der Untertänigkeit, Freiheit zum eigenen Entwurf, zur Gestaltung der Zukunft“ (Dessauer 1959, S. 184).

Entlastung schließt die Fürsorge durch Andere am Anfang und am Ende des Lebens ein, aber auch die Fürsorge im Falle einer Behinderung oder von Krankheit, die vorübergehend die Handlungsfähigkeit einschränkt. Das Entlastungsprinzip erlangt angesichts der benannten sozialen Probleme im Alter eine besondere Relevanz. Der fürsorgliche Umgang mit Alter, Behinderung und Krankheit kann in einer an Funktionalität und Effizienz orientierten Gesellschaft nur gewährleistet werden, wenn alle technischen Entlastungsressourcen genutzt werden. Die Frage ist aber, wann diese technisch disponierte gesellschaftliche Fürsorglichkeit in paternalistische Effekte bzw. in Entmündigungen umschlägt, wann sie also zu etwas, den Menschen in seinen Vermögen und seinem Selbstverständnis Bedrohendem wird.

\section{Autonomie, Würde und Subsidiarität als Prinzipien des Selbst- und Gesellschaftsverständnisses}

Schauen wir auf drei, unser Selbst- und Gesellschaftsverständnis leitende Ideen, und zwar die der Autonomie, der Würde und der Subsidiarität, die Bedingungen formulieren, unter denen der Einsatz assistiver Systeme rechtfertigbar ist. Erinnern wir zunächst an einige elementare kantische Grundlagen der Autonomieidee. Autonomie als Grundbegriff eines aufgeklärten Selbst- und Gesellschaftsverständnisses bedeutet, dass der Einzelne prinzipiell imstande ist, kraft seines Vernunftvermögens seine Alltagsgeschäfte ohne Bevormundung durch andere zu erledigen und alle Entscheidungen, die ihn, seine Lebensweise und sein Verhältnis zur Gesellschaft betreffen, selbst zu treffen. Das Vernunftvermögen des Menschen schließt ein, dass diese Entscheidungen nicht auf Kosten anderer gehen, also deren Autonomie durch die eigene nicht gefährdet ist. Es besteht demnach kein Widerspruch zwischen einer individuellen und einer gesellschaftlichen Entlastung. Die gesellschaftliche Komponente impliziert aber auch das Absehen von eigenen Interessen um des Ganzen willen. Natürlich ist die Autonomie ein Ideal, eine Orientierungsidee, die in einer komplexen und kompetenz- teiligen Gesellschaft nie erreicht werden kann. Ist nun die Autonomie des Einzelnen eingeschränkt, ist die Gesellschaft verpflichtet, das Individuum entweder durch Erziehung zur Autonomie zu führen, oder aber es so zu unterstützen, dass es so weit wie möglich imstande ist, seine Geschäfte selbständig zu erledigen. Kann die Autonomie aufgrund körperlicher oder geistiger Einschränkungen nicht mehr dauerhaft erlangt werden, soll zumindest die Würde des Menschen gewahrt bleiben.

Die Würdeidee ist mit der der Autonomie verknüpft und bedeutet, dass der Mensch immer so behandelt werden soll, als wenn er autonom wäre. Das autonome Subjekt ist Selbstzweck und niemals Objekt des Handelns. Es muss auch in Zeiten seiner physischen oder geistigen Ohnmacht so behandelt werden, dass niemals der Wert seines Lebens infrage steht, sondern dessen Einzigartigkeit und moralischer Status im Fokus bleiben. Würde zeichnet sich dadurch aus, dass sie sich der Verhandelbarkeit entzieht. Über Werte können wir verhandeln, über die Würde nicht.

Aus den Leitideen der Autonomie und der Würde des Menschen lässt sich in gewisser Weise die Idee der Subsidiarität - eine Schlüsselkategorie der katholischen Soziallehre - deduzieren. Ein autonomes Wesen darf nicht bevormundet werden, auch wenn diese Bevormundung den ökonomischen Interessen einer Gesellschaft entgegenkommt. Ihm darf allerdings auch nichts abgenommen werden, was es selbst entscheiden und leisten kann. Das Subsidiaritätskonzept ist zwar an die Ideen der Autonomie und Würde des Individuums geknüpft, sein Anspruch gilt aber für die gesamte demokratische Organisation der Gesellschaft. Alles, was auf der jeweils niedersten Stufe der Gesellschaftsorganisation geregelt werden kann, soll auch dort geregelt werden.

\section{Zur Ambivalenz von Entlastung und Entmündigung}

Die Frage ist nun, wann eine Unterstützungsmaßnahme in die Autonomie des Unterstützten eingreift, wann sie dessen Würde gefährdet und mit dem Prinzip der Subsidiarität kollidiert?

Verengen wir zunächst das Feld der Erörterung: Es sollen keine Fälle diskutiert werden wie die apparative Lebensverlängerung komatö- 
ser Patienten oder Fälle schwerer Demenz. Hier erübrigt sich die Frage nach der Autonomie. Es geht hier nicht um Assistenz, sondern um die Frage nach der menschlichen Würde in der unwiederbringlich letzten Phase des Lebens.

Da die Grenze zwischen Systemen, die Gesunde entlasten, und solchen, die Kranke, Behinderte und Alte unterstützen, nicht klar gezogen werden kann, werden viele Applikationen Behinderten und Nichtbehinderten, Gesunden und Kranken zur Verfügung stehen, was die Nutzung sowohl in ökonomischer als auch sozialer Hinsicht vereinfachen könnte, da wir an die Unterstützung schon in gesunden Zuständen gewöhnt wurden. Die Systeme agieren aufgrund permanenter, und - je nach Umfang der Dienstbarkeit - möglichst umfassender extra- und intrakorporaler Observation des Nutzers. Beeinflussungen und sanfte Steuerungen durch das System sollen eine optimale Abstimmung individueller und allgemeiner Interessen ermöglichen. Dies kann dadurch geschehen, dass bestimmte Handlungsoptionen durch das System nicht angeboten werden. Wenn assistive Systeme so eingerichtet werden, dass es ein Primat der Wirtschaftlichkeit gibt, kann dies zu einer Kaschierung inhumaner gesellschaftlicher Verhältnisse führen, schließlich repräsentiert das System Gesamtorganisation und Werthierarchie der Gesellschaft. Wenn Technik dem Menschen dienen, nicht aber der Mensch ökonomischen Intentionen und Sachzwängen unterworfen sein soll, muss sich ihr Einsatz durch die genannten Leitideen begrenzen lassen. Dies ist eine Herausforderung, bedeutet es doch auch eine Begrenzung technischer Ansprüche, und fordert dies zu Ausdifferenzierungen der Technik heraus. So müssten etwa gewisse Adaptivitätsansprüche, die sich im Verschwinden von Schnittstellen in der Mensch-System-Interaktion artikulieren, aufgegeben werden. Was sich nicht bemerkbar macht, kann vom Nutzer weder kontrolliert, noch gesteuert werden. Problematisch sind auch Ansprüche, die im Bereich des Biocybernetic Computing formuliert werden, wo der Mensch eng angelehnt an deterministische Konzepte der Gehirnforschung als Maschine konzipiert wird. Unabhängig davon, ob diese Konzepte modernen Erkenntnissen der Naturwissenschaft entsprechen, sind sie nicht mit der Autonomieidee in Einklang zu bringen. Auch wenn sich moderne Systemtech- nologien nicht auf ihren Werkzeugcharakter reduzieren lassen, wäre der komplette Verlust dieses Charakters eine Entmachtung und Entmündigung des Menschen. Die angestrebte Autonomie des Systems würde der des Nutzers widerstreiten.

Die Ausrichtung der Systemtechnologie an ökonomischen Zwecken und technischen Machbarkeitsphantasien kann zu Entmündigungen führen, denn es ist nicht immer effizient, was der Einzelne oder die Gesellschaft entscheidet, aber es kann Ausdruck eines humanen Gesellschaftsverständnisses sein, in dem Alte nicht nach ihrer Leistungsfähigkeit beurteilt werden und Kultur als Verzögerungsphänomen gepflegt wird, auch wenn sich diese Pflege nicht „rechnet“. Es ist nicht zuletzt Ausdruck der Kultur, dass sie Sphären eines auf Effizienz ausgerichteten ökonomischen Handelns begrenzt.

Das Zauberlehrlingsproblem, das sich in einer sich verselbständigenden Technik artikuliert, hat uns eingeholt und stellt bei aller Entlastung eine Bedrohung für unser Selbstverständnis dar. Viele Entscheidungen, die wir in einer informationstechnologisch hoch erschlossenen Gesellschaft treffen, müssen in ihren Auswirkungen mitverantwortet werden.

Konkretisieren wir die Ambivalenz von Entlastung und Entmündigung. Entlastung findet statt, wo verlorene Handlungsmöglichkeiten substituiert oder eingeschränkte wiederhergestellt werden. Entlastung ist aber kein Selbstzweck. Ein völlig entlasteter Mensch hätte nichts mehr zu entscheiden, hätte sein Leben nicht mehr zu führen, hätte also als moralisches Subjekt abgedankt. Da alles $\mathrm{zu}$ seinem vermeintlich Besten eingerichtet ist, hätte er keinerlei Verantwortung für sein Tun und Lassen zu tragen. Es geht also im Falle der Entlastung um die Fragen „Entlastung wozu?“, „Entlastung von was?" und „Wann ist die Würde des Menschen durch das technische Entlastungsprinzip gefährdet?“. Letzteres ist dann der Fall, wenn der Nutzer eine Reduzierung auf eine Rolle erfährt. Pflege- und Unterstützungsmaßnahmen dienen dem Zweck, die physiologische und psychologische Funktionalität zu wahren oder zu verbessern, um möglichst eigenständig handeln zu können. Es geht bei Assistenzsystemen also nicht um Substitutionen von Entscheidungen und Handlungen, sondern um die Ermöglichung eigenständigen 
Handelns. Assistive Systeme sollten in diesen Bereichen anders ausgelegt sein als im medizinischen und therapeutischen Bereich. Es geht auch nicht um einen automatischen Co-Piloten, der im Notfall anstelle des Piloten agieren kann. Das assistive System ersetzt nicht die Entscheidungs- und Handlungsfähigkeit des Nutzers, sondern dient dem Zweck, diese soweit wie möglich wiederherzustellen. Auch wenn dies nicht mehr völlig gelingt, bleibt sie der Maßstab für den Einsatz assistiver Systeme. Diese nehmen Nutzer zwar immer in Rollen wahr, ein Problem entsteht aber, wenn die Rollenfestlegung keinen Entscheidungs- und Handlungsspielraum für den Nutzer lässt und die Rolle für die ganze Person steht. Die durch assistive Systeme angestrebte Entlastung darf also nicht zu einer Enteignung von Vermögen führen.

Entmündigung findet statt, wo jemand ,nur“ noch in seiner Rolle als Kranker, Behinderter oder Alter wahrgenommen wird. Überall, wo dem Unterstützten Wahloptionen vorenthalten werden, wohlgemerkt Wahloptionen, die überschaubar sind - schließlich kann ein Überangebot von Optionen Handlungsbereitschaft auch einschränken -, findet ein erster Schritt zur Entmündigung statt, auch wenn diese Vorenthaltung zu seinem vermeintlich Besten geschieht. Solange von der Autonomie einer Person ausgegangen wird, muss auch von Entscheidungen gegen das Gesundheitsprinzip, möglicherweise um eines anderen Gutes willen, möglicherweise aber auch um einer kurzfristigen Lust willen, ausgegangen werden. Ein autonom gedachtes Wesen kann nun einmal wider sein vermeintlich Bestes handeln.

Entmündigung findet statt, wenn noch nicht einmal ein zeitweiliger Systemausstieg mehr gewährleistet ist. Gewiss ist dies ein heikler Punkt, denn ein auch nur kurzfristiger Systemausstieg kann fatale Folgen haben. So kann eine vorübergehende Einschränkung der Mündigkeit eher den längerfristigen Intentionen des Nutzers entsprechen als eine bedingungslose Anerkennung seiner Mündigkeit. Nicht immer ist die situative Laune das, was den Interessen des Individuums längerfristig zuträglich ist. Systemkontrolle und -eingriff können aus verschiedenen Gründen nicht immer gewährleistet sein, da 1) der Nutzer in der Regel Laie ist, der außerstande ist, das System zu kontrollieren bzw. zu steuern - und je komplexer und leistungsstärker die Systeme werden, desto mehr muss von einer Unkontrollierbarkeit durch den Laien ausgegangen werden; da 2) der Nutzer aufgrund von Krankheit, Altersschwäche oder Behinderung nicht imstande ist, Kontrollen und Steuerungen vorzunehmen; und da 3) der Nutzer aufgrund zeitlich begrenzter Bedienungsunfähigkeit nicht zu Kontrollen und Steuerungen imstande ist. Diese Fälle sind zu berücksichtigen, stellen aber an sich noch keine Entmündigung dar, solange die Systeme eine Option zur Kontrolle und Steuerung durch einfache Bedienung bieten. Es müssen Systemfunktionen angezeigt werden können und abstellbar sein. Ein vorübergehender Ausstieg aus der Systemunterstützung muss gewährleistet sein, wenn die Unterstützung nicht überlebensnotwendig ist.

Entmündigungsgefahren sind v. a. da zu sehen, wo Systeme den Anspruch erheben, die Stimmungs- und Gefühlslage sowie die ,eigentlichen" Intentionen einer Person zu erfassen. In diesem Fall findet eine metaphysische Aufladung der Systeme statt. Es wird der Anspruch erhoben, den Menschen wie eine Maschine kausal erklären zu können. Es ist evident, dass deterministische Ansprüche mit dem kantischen Konzept des Menschen als Bürger zweier Welten nicht vereinbar sind. Autonomie und Würde sind in der Sphäre der noumenalen Welt angesiedelt, können also deterministisch nicht gefasst werden.

\section{Konsequenzen zur Vermeidung von Entmündigungen durch Assistenz- und Pflegesysteme}

Formulieren wir abschließend Konsequenzen zur Vermeidung von Entmündigungen: Assistive Systeme müssen so ausgelegt sein, dass sie die Autonomie des Nutzers fördern, wobei diese eine relative Größe ist, die an tatsächliche körperliche und geistige Möglichkeiten angemessen sein muss. Prinzipiell müssen Systeme, jenseits ihrer Funktion, Leben zu erhalten und vor Gefährdungen zu bewahren, dem Nutzer Handlungsoptionen sowie Möglichkeiten zeitweiligen Ausstiegs aus der Systemunterstützung gewähren. Dies mag mit Risiken verbunden sein, die es aber zu ertragen gilt, schließlich hängt an ihnen unser Selbst- und Gesellschaftsverständnis. Entscheiden können 
wir nur, was die Möglichkeit birgt, auch falsch zu sein. Die Vision einer autonomen Technologie als unser stiller Begleiter und Beschützer birgt übrigens nicht nur Risiken, sondern auch einen logischen Widersinn. Ein wirklich autonomes System wäre freilich nicht mehr unser Werkzeug und würde uns auch Dienste verweigern. Angesichts der konstatierten Ambivalenz assistiver Systeme zur Unterstützung von Alten und Pflegebedürftigen gilt es, frühzeitig die Systementwicklung kritisch zu begleiten. Es müssen Rahmenbedingungen für die Systementwicklung gegeben werden, in denen die Grundprinzipien unseres Selbstund Gesellschaftsverständnisses, die Autonomie, die Würde und die Subsidiarität ausformuliert werden. Moderne Technologie hat ein Grad der „Autonomie“ erreicht, das diese Grundprinzipien infrage stellen kann. Der Mensch kann dies nicht hinnehmen, solange er diese Prinzipien als Bedingungen seiner Existenz begreift.

\section{Literatur}

Dessauer, F., 1959: Der Streit um die Technik. Freiburg i. B.

Gehlen, A., 1993: Der Mensch. Seine Natur und seine Stellung in der Welt, Frankfurt a. M (Erstveröffentlichung 1940)

Kapp, E., 1877: Grundlinien einer Philosophie der Technik. Zur Entstehungsgeschichte der Cultur aus neuen Gesichtspunkten, Braunschweig

\section{Kontakt}

Prof. Dr. Klaus Wiegerling Institut für Technikfolgenabschätzung und Systemanalyse (ITAS)

Karlsruher Institut für Technologie (KIT)

Karlstraße 11, 76133 Karlsruhe

Tel.: +49 721 608-23839

E-Mail: klaus.wiegerling@kit.edu

\section{Autorenhinweise}

Wir bitten alle Autorinnen und Autoren, die ein Manuskript bei TATuP einreichen, die folgenden Hinweise zu beachten:

Umfang: Eine Druckseite umfasst max. 3.500 Zeichen (ohne Leerzeichen). Für den Umfang eines Beitrags ist die Rubrik, in der er erscheint, ausschlaggebend. Genauere Angaben erhalten die Autoren von der Redaktion.

Abstract: Autoren, deren Beiträge im Themenschwerpunkt des Heftes oder in den Rubriken TA-Konzepte und -Methoden und Diskussionsforum sowie TA-Projekte erscheinen, werden gebeten, ihrem Beitrag ein Abstract voranzustellen, in dem eine kurze inhaltliche Übersicht über den Beitrag gegeben wird. Die Länge dieses Abstracts sollte 780 Zeichen (ohne Leerzeichen) nicht überschreiten.

Abbildungen, Diagramme und Tabellen: Abbildungen und Tabellen sind sowohl in das eingereichte Manuskript einzufügen sowie auch getrennt von der ersten Fassung des Manuskripts einzusenden. Abbildungen und Tabellen bitte mit Überschrift und Quellenangabe versehen. Wurden sie vom Autor selbst erstellt, bitte die Formulierung ,eigene Darstellung“ als Quellenangabe verwenden Zum Format: Tabellen sind als Word-Datei, Diagramme in Excel und Abbildungen in Adobe Illustrator oder Powerpoint zu liefern. Sollten Sie lediglich andere Formate zur Verfügung haben, wenden Sie sich bitte frühzeitig an die Redaktion. Aus Gründen der Seitenplanung und des Layouts liegt die Entscheidung über die endgültige Größe und Platzierung der Abbildungen und Tabellen innerhalb des Beitrags bei der Redaktion.

Bibliografische Angaben: Die zitierte Literatur wird am Ende des Beitrags als Liste in alphabetischer Reihenfolge angegeben. Im Text selbst geschieht dies in runden Klammern (z. B. Wiegerling 2011); bei Zitaten ist die Seitenangabe hinzuzufügen (z. B. Fink/Weyer 2011, S. 91). Bei den Angaben in der Literaturliste orientieren Sie sich bitte an folgenden Beispielen:

Monografien: Wiegerling, K., 2011: Philosophie intelligenter Welten. München

Bei Aufsätzen: Fink, R.D.; Weyer, J., 2011: Autonome Technik als Herausforderung der soziologischen Handlungstheorie. In: Zeitschrift für Soziologie 40/2 (2011), S. 91-111

Bei Beiträgen in Sammelbänden: Mehler, A., 2010: Artifizielle Interaktivität. Eine semiotische Betrachtung. In: Sutter, T.; Mehler, A. (Hg.): Medienwandel als Wandel von Interaktionsformen. Heidelberg

Bei Internet-Quellen: Waterfield, J., 2006: From Corporation to Transnational Pluralism. London; http:// www.plugin-tot.com (download 12.3.09) 